\title{
LIVER
}

\section{Uncovering the secrets of secretin}

New research shows that increased secretin signalling, in addition to stimulating cholangiocyte proliferation, also plays a part in the pathogenesis of liver fibrosis associated with cholestasis.

Cholestatic liver diseases, such as primary sclerosing cholangitis (PSC), are characterized by fibrosis in intrahepatic and extrahepatic bile ducts and progressive liver pathology. Cholestasis results in cholangiocyte proliferation, a process thought to be involved in the development of hepatic fibrosis in these diseases.

In previous research, secretin, a neuropeptide hormone that binds the secretin receptor, was shown to be expressed in the liver only by cholangiocytes; moreover, expression of secretin and the secretin receptor by cholangiocytes was upregulated in response to cholestasis. "We demonstrated that secretin produced by cholangiocytes and $\mathrm{S}$ cells in the duodenum reduces the expression level of the microRNAs let-7a and miR-125b, resulting in an upregulation of target genes known to be proliferative or profibrotic factors for cholangiocytes," says author Shannon Glaser. "However, it was unclear if secretin also played a role in the pathogenesis of hepatic fibrosis."

In the new study, the investigators used two mouse models of cholestatic liver disease to investigate secretin signalling: wild-type mice that had undergone bile-duct ligation (BDL); and Mdr2 (also known as Abcb4) double knockout mice. These models develop severe cholestatic liver disease and extensive liver fibrosis. By treating these mice with either secretin or a secretin receptor antagonist (sec 5-27), the researchers were able to probe how secretin signalling affects cholestatic liver disease.

Treatment of wild-type and BDL mice with secretin increased the degree of histologically assessed liver fibrosis; by contrast, liver fibrosis was reduced in $\mathrm{BDL}$ and $\mathrm{Mdr2}^{-/-}$mice treated with secretin antagonist compared to untreated mice. Moreover, although $\mathrm{Mdr}^{-/-}$and BDL mice had increased hepatic mRNA expression of transforming growth factor $\beta$ (Tgfb1) and fibronectin (Fn- 1$)$ compared with wild-type mice, expression of these genes was reduced in mice treated with secretin receptor antagonist. Levels of SMAD2 and SMAD3 phosphorylation, which are indicative of TGF $\beta 1$ signalling, were increased in wild-type mice treated with secretin and $\mathrm{Mdr2}^{-/-}$mice, whereas antagonism of secretin signalling resulted in lower SMAD2 and SMAD3 phosphorylation levels.

The researchers also characterized the secretin signalling axis in liver tissue samples from patients with PSC. Compared with samples from healthy controls, liver tissue from patients with PSC had

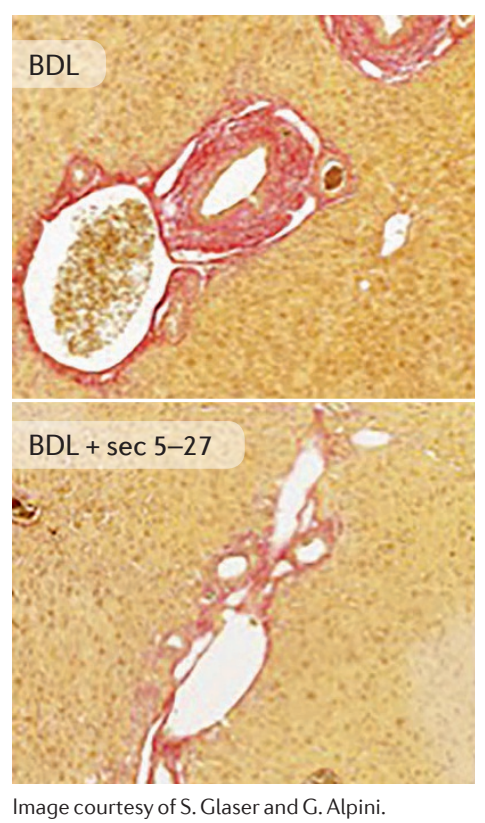

increased expression of secretin and the secretin receptor, as well as TGF $\beta 1$, the TGF $\beta 1$ receptor and markers of fibrosis.

"Targeting the secretinsecretin-receptor axis or the let-7a pathway might provide a therapeutic approach for the treatment of human cholangiopathies, including PSC," concludes study author Gianfranco Alpini.

Hugh Thomas

ORIGINAL ARTICLE Wu, N. et al. The secretin/ secretin receptor axis modulates liver fibrosis through changes in TGF- $\beta 1$ biliary secretion. Hepatology http://dx.doi.org/10.1002/hep.28622 (2016) 